\title{
Resection of large epidermoid tumors ventral to the brainstem: techniques to expand the operative corridor across the basilar artery
}

\author{
Aaron A. Cohen-Gadol, M.D., M.Sc. \\ Goodman Campbell Brain and Spine, Department of Neurological Surgery, Indiana University School of \\ Medicine, Indianapolis, Indiana
}

Epidermoid tumors comprise about $1 \%$ of all intracranial tumors. They are congenital lesions that arise from paramedian cisterns within the posterior fossa. These tumors present as heterogeneous hyperintense lesions on FLAIR and homogenous hyperintense lesions on DWI. Surgical resection remains the most accepted form of therapy, but epidermoid tumors may recur. These tumors are well exposed through a traditional retrosigmoid approach. The tumor can be removed relatively easily as it is avascular. However, the propensity of this tumor type to fill the small spaces within basal cisterns and attach to cranial nerves may make its complete resection challenging. Tumors resection has to preserve the surrounding arachnoid membranes encasing the cranial nerves.

The author presents the case of a 42-year-old woman with a 1-year history of imbalance and nystagmus. An MRI revealed a large right-sided CP angle epidermoid tumor filling the ventral brainstem cistern and extending to the contralateral side, compressing the brainstem. The accompanying video illustrates resection of this mass through an extended (exposing the sigmoid sinus) retrosigmoid approach. The author removed the tumor piecemeal while protecting the cranial nerves. Small pieces of affected arachnoid covering the cranial nerves were not significantly manipulated. To excise the tumor along the contralateral paramedian cistern, the author used the space between the $\mathrm{V}$ and VII/VII cranial nerves to expose the space contralateral to the basilar artery and remove additional tumor. This maneuver allowed gross total resection of the tumor without a need to employ a more elaborate skull base approach such as petrosectomy. At 3-month follow-up visit after surgery, the patient's neurological exam returned to normal.

The video can be found here: http://youtu.be/CzRb-GUvhog.

(http://thejns.org/doi/abs/10.3171/2014.V1.FOCUS13308)

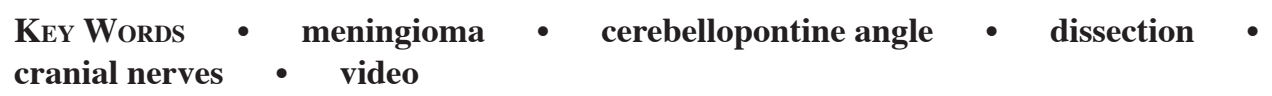

Manuscript submitted July 25, 2013.

Accepted November 6, 2013.

Please include this information when citing this paper: DOI: 10.3171/2014.V1.FOCUS13308.

Address correspondence to: Aaron A. Cohen-Gadol, M.D., M.Sc., Goodman Campbell Brain and Spine, Department of Neurological Surgery, Indiana University School of Medicine, 355 West 16th Street, \#5100, Indianapolis, IN 46202. email: acohenmd@gmail.com. 